\title{
Applying Hybrid Decision-Making Method Based on Fuzzy AHP-WOWA Operator for Emergency Alternative Evaluation of Unattended Train Operation Metro System
}

\author{
Bobo Zhao, ${ }^{1}$ Tao Tang, ${ }^{2}$ and Bin Ning $^{2}$ \\ ${ }^{1}$ National Engineering Research Center of Rail Transportation Operation and Control System, Beijing Jiaotong University, \\ No. 3 Shang Yuan Cun, Haidian District, Beijing 100044, China \\ ${ }^{2}$ State Key Laboratory of Rail Traffic Control and Safety, Beijing Jiaotong University, No. 3 Shang Yuan Cun, Haidian District, \\ Beijing 100044, China
}

Correspondence should be addressed to Bobo Zhao; bbzhao@bjtu.edu.cn

Received 27 October 2015; Accepted 17 December 2015

Academic Editor: Konstantinos Karamanos

Copyright (C) 2016 Bobo Zhao et al. This is an open access article distributed under the Creative Commons Attribution License, which permits unrestricted use, distribution, and reproduction in any medium, provided the original work is properly cited.

Optimal alternative selection to address the emergency situation is critical for dispatcher group in Unattended Train Operation (UTO) to guide emergency process. It is difficult to provide the precise decision value under one criterion and to evaluate the emergency alternatives among multiple dispatchers. This paper presents a hybrid emergency decision-making method integrating fuzzy analytic hierarchy process (FAHP) described by linguistic terms with enhanced weighted ordered weighted averaging (WOWA) operator. The enhanced WOWA operator aggregates the preference matrices of multidispatcher through the constructed emergency response task model of dispatcher group in OCC. This calculation approach takes into consideration the relations of emergency tasks to derive the importance weights of dispatchers and integrates them into the ordered weighted averaging (OWA) operator weights based on a fuzzy membership relation. A case study of applying the method in an emergency of a train fire is given to demonstrate the feasibility and usefulness of the methods associated with the group multicriteria decision-making (GMCDM) theory in emergency management of UTO metro system.

\section{Introduction}

Unattended Train Operation (UTO) refers to the automated metro system in which trains run fully automatically without any operating staff onboard [1]. For the advantages such as cost-effectiveness, high traffic frequency, and flexibility, the UTO metro has a worldwide application spread as a global adoption trend. Researches on UTO metro have been mostly concentrated on how to operate the trains safely and timely and the studies on decision support for the emergency situation of UTO system have spread scarcely.

Nevertheless, there have been many researches on decision support for decision makers in emergency response system in other domains. The integrated and comprehensive real-time on-line decision support system (RODOS) was designed to provide off-site emergency management in the event of a radiation accident in nuclear emergencies [24]. The method of multicriteria decision analysis (MCDA) was integrated into RODOS to evaluate alternatives or countermeasure strategies within emergency and remediation management $[5,6]$. Other decision support systems also were presented to manage emergency response operations for large scale industrial accidents such as hazardous materials emergencies $[7,8]$.

Besides, several studies have investigated on the emergency response system by modelling the emergency response task network. Abrahamsson et al. [9] provided a better framework in which an emergency response system could functionalize during a specific operation and helped to identify the potential events and/or circumstances, which 
could significantly affect the performance of the emergency response system. Using task network mapping and analysis, Wang et al. [10] presented a method of improving the performance of emergency response system by taking the time factor into consideration. Modelling the emergency response task network is an effective way for decision support in emergencies. However, these studies overlooked that people are the ultimate decision makers who are responsible for dealing with the emergency accidents and minimizing the casualties and property loss.

Moreover, some researchers studied on the effect of human factors under emergency situation in UTO metro system. For example, Wang and Fang [11] presented a structured procedure to analyze the error behaviors of traffic dispatcher in emergencies based on the human information processing theory and the modified task analysis framework. Karvonen et al. [12] analyzed the requirements of metro drivers to reveal what should be provided to compensate for the absence of driver in UTO metro system. However, these papers only studied the effect of human factors on single type position such as traffic dispatcher or driver, rather than group decision for metro emergencies. The challenges of UTO metro operation are, without driver onboard, how the abnormal situation could be found out and be restored to the normal operations and this indicates that the Operating Control Center (OCC) should ensure the detection and management of emergency situations [13] and take proper emergency responses and clearance to avoid further damage.

In case of any emergency in UTO metro, the emergency response process is to immediately organize the related agencies, raise and dispatch various resources, and develop and carry out the emergency response plans, with the goal of minimizing casualty and losses caused by disasters [14]. The crucial point is how the dispatchers differentiate the distinct and real information and make the proper decision. Such decision making is an expertise balancing process within a number of criteria and opinions from different dispatchers, who have different knowledge about emergency alternatives and make different contributions to different emergency alternatives [15]. Sometimes, the opinions from different dispatchers' conflict with each other and, therefore, emergency alternative evaluation in UTO metro system is a typical group multicriteria decision-making (GMCDM) problem. GMCDM is an evaluation approach to aggregate the information of alternatives to obtain the best solution for emergency decision makers [16].

Saaty [17] proposed a method named analytic hierarchy process (AHP), which provided a pairwise comparison matrix for decision makers to express their preferences regarding multiple criteria and alternatives. This method is widely used for solving multicriteria decision-making problems including planning, selecting a best alternative, resource allocation, and resolving conflicts [18]. Nevertheless, the AHP method is often criticized for its inability to incorporate the inherent uncertainty and imprecision associated with mapping the decision maker's perceptions to exact numbers [19]. To solve this problem, the fuzzy analytic hierarchy process (FAHP) used fuzzy preference relations to incorporate the ambiguities and uncertainties that usually exist in human judgment generally $[20,21]$. The FAHP, adopting the concepts of fuzzy set theory and hierarchical structure analysis, has been widely employed to solve the alternative selection and justification problem in different research areas [22-25].

The ordered weighted averaging (OWA) operator proposed by Yager [26] provided the selection of various preferences of decision makers from the optimistic view to the pessimistic one [27]. However, it did not consider importance weights through OWA operator. Actually, the weighted mean cannot be described by the arithmetic mean of OWA aggregations [28]. To solve this problem, Torra [29] proposed the weighted OWA (WOWA) aggregation which incorporated the important weighting into the OWA operator. Since WOWA operator was proposed, it has been widely applied in the fields of multicriteria decision-making $(\mathrm{MCDM})$ system and metadata aggregation problems [30, 31].

Great efforts have been made to solve GMCDM problems in UTO emergencies. In this paper, we adopted the method of FAHP to solve the decision support under multicriteria and employed enhanced WOWA operator to aggregate each decision from different decision makers, in which the weight assigned to operator was one key factor for the performance of the alternatives selection.

The remainder of the paper is organized as follows. First, an overview of UTO system in China is given. Second, FAHP method and WOWA operator are introduced. Third, a hybrid method, combining FAHP and the enhanced WOWA operator weights, is specified. This method integrates the weights applied to represent the significance level associated with the importance of dispatchers in a scenario of emergency response task system for UTO metro system. Then it orders weights into the enhanced WOWA operator and connects the operator with the concept of fuzzy logic in a hierarchy pattern under the different knowledge situation of dispatcher group. Fourth, a case study is presented to demonstrate how the proposed method is applied to obtain the sequence order of emergency alternatives in case of train fire occurring between stations. Finally, some conclusions are drawn.

\section{UTO System}

We adopted the Line YanFang in Beijing as an investigation target, which is under construction and will become the first UTO line regulated in automated operation in Beijing. Furthermore, we investigated Line 10 in Shanghai, in which the organization has been designed for operational service of UTO system. Based on the learning experience of Line 10 in Shanghai, we attended the designation and construction process of the Line YanFang and discussed the new challenges in UTO metro, which should be understood fully in future operation.

The characteristics of UTO system under emergency environment are that the dispatchers in OCC cannot reach available personnel to obtain the contingent factors of emergency situation remotely in a short time. The factors include the location, the scope of emergency, and the passenger emotion under emergency. Without personnel in train, any major functional failures or emergencies from the operated 
TABLE 1: Detailed responsibilities of dispatchers in OCC.

\begin{tabular}{ll}
\hline Different types of dispatchers & Responsibility \\
\hline Traffic dispatcher & Managing and supervising the operation such as daily timetable, train service, and train operation. \\
Environment dispatcher & Managing and supervising electromechanical devices, water supply, and drainage system. \\
Power dispatcher & Managing and supervising third rail power supply devices. \\
Passenger dispatcher & Supervising the passengers onboard and providing passengers with service under emergency. \\
Vehicle dispatcher & Supervising the status of equipment onboard and handling the exceptional situation onboard remotely. \\
Maintenance dispatcher & Receiving the failure and assigning the maintenance task. \\
\hline
\end{tabular}

train are not easy to be handled quickly, and the passengers have to be supplied remote service or guidance of station staff with some delay. As a result, the OCC becomes the initial and primary responding organization to emergency situation. The major responsibilities of dispatchers in OCC are to capture the characteristics of emergency situation and to apply appropriate recovery process, which involves removing the passengers in stranding train, locating the equipment failure in system, monitoring and responding the alarm from the different sensors, and so forth. It could be sequence order of EMT (Executive Management Team) application and need sophisticated automatic techniques without driver onboard.

The organization of metro faces a big challenge to restore the emergency situation and to supply safe and flexible operational services for passengers. For this reason, the simultaneous efforts of organization training for adapting to a new pattern of emergency have been done with the development of UTO metro system. The dispatchers should be assigned the corresponding reasonable responsibility with respect to different elements in the UTO metro. The elements include the passengers, train, signaling system, station, and infrastructure. In order to reveal the interdependence among these elements and the management pattern of OCC organization, we investigated Line 10 in Shanghai to learn the organization pattern and management content of UTO metro, and then we designed OCC's organization with six types of dispatcher role in the YangFang line of Beijing, represented by traffic dispatcher, environment dispatcher, power dispatcher, passenger dispatcher, vehicle dispatcher, and maintenance dispatcher, respectively. The detailed responsibilities of dispatchers from the role-function are summarized in Table 1.

From Table 1, the new obligation of dispatcher is to compensate for the absent responsibility of driver. In conventional metro, the driver has to participate, observe, interpret, and react to emergency events in the surrounding situation [12]. From this point of view, taking care of the passengers and handling the exceptional events onboard are the hidden tasks for the driver to execute, and, compared with the conventional actor group, the organization in OCC should supply new job positions to compensate for the indispensable parts of operation service. Therefore, the added dispatchers are named as passenger dispatcher and vehicle dispatcher, respectively, which replace the driver to provide emergency service for the passenger and handle exceptional situation onboard.

\section{Methods}

3.1. FAHP. Based on the pair-by-pair comparison values for a set of alternatives, FAHP is applied to elicit a corresponding priority vector that represents preferences [32] and is capable of capturing a human's appraisal of ambiguity when complex multicriteria decision-making problems are considered [33]. Fuzziness and vagueness are the characteristics of emergency decision-making problems $[34,35]$ and FAHP can be able to tolerate vagueness or ambiguity in the emergency decisionmaking process.

The basics about the fuzzy preference relations employed in FAHP are the following: let $X=\left\{x_{1}, x_{2}, \ldots, x_{n}\right\}(n \geq 2)$ be the set of alternatives. A fuzzy preference relation $R$ on a set of objects $X$ is a fuzzy set on the product set $X \times X$, and it is characterized by a membership function $\mu_{R}: X \times X \rightarrow[0,1]$. The preference relations can be expressed by $n \times n$ matrix $R=\left(r_{i j}\right)_{n \times n}$, and $r_{i j}$ is interpreted as the preference degree or intensity of the alternative $x_{i}$ over $x_{j}$. The $r_{i j}$ has the following characteristics:

(1) $r_{i j}=0.5$; it indicates indifference between $x_{i}$ and $x_{j}$ $\left(x_{i} \sim x_{j}\right)$;

(2) $0 \leq r_{i j}<0.5$; it indicates that $x_{j}$ is strictly preferred to $x_{i}\left(x_{j}>x_{i}\right)$

(3) $0.5<r_{i j} \leq 1$; it indicates that $x_{i}$ is strictly preferred to $x_{j}\left(x_{i}>x_{j}\right)$. In particular, $r_{i j}=1$ denotes that $x_{i}$ is definitely preferred to $x_{j}$.

In general, the matrix $R=\left(r_{i j}\right)_{n \times n}$ is called a reciprocal fuzzy preference relation (FPR), such that $r_{i i}=0.5$ and $r_{i j}+$ $r_{j i}=1$, for all $i, j=1,2, \ldots, n$.

Definition 1 (see [20]). A reciprocal fuzzy preference relation $R=\left(r_{i j}\right)_{n \times n}$ is additive consistency, if, for all $i, j, k=1,2, \ldots, n$ with $i<k<j$, the condition $r_{i k}+r_{k j}=r_{i k}+0.5$.

The additive consistency of fuzzy preference relation is a strong condition to assess whether the decision-making judgment is consistent. In this study, the matrix $R$ is always assumed to be reciprocal fuzzy preference relation and to have the property of additive consistency.

3.2. WOWA Operator. The WOWA operator takes two aspects of weighting vectors into consideration: the importance weights $p$ and OWA operator weights $w$. 


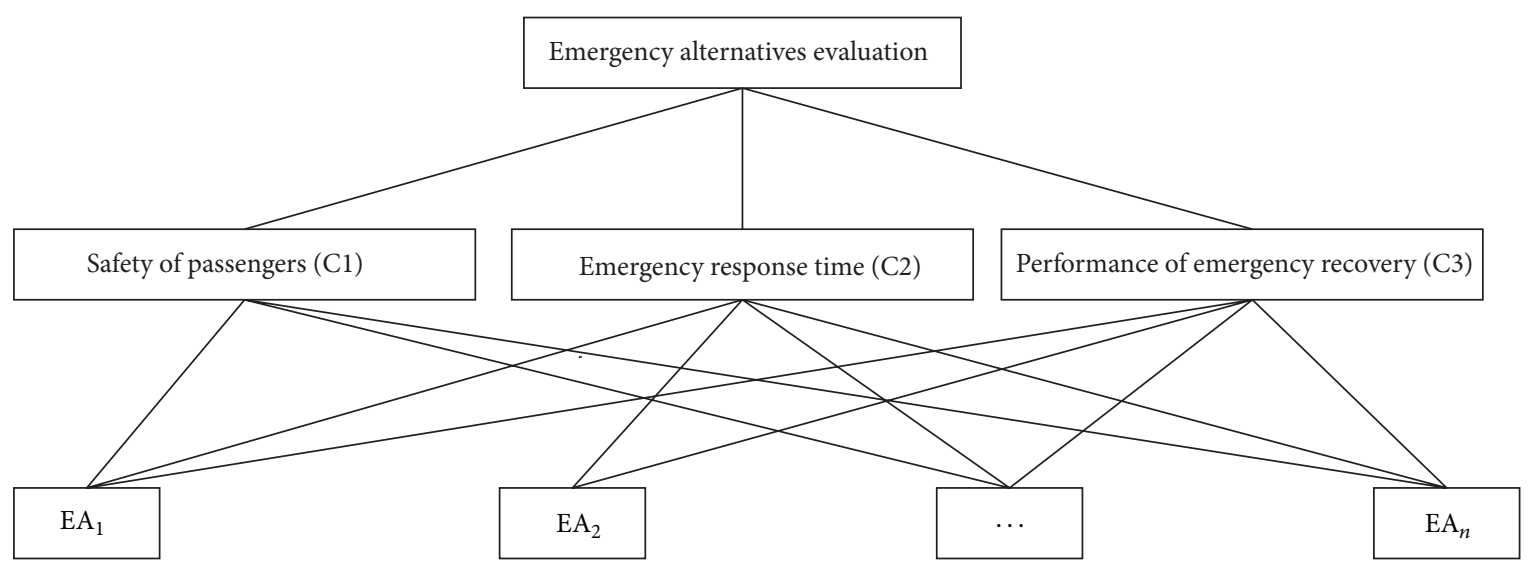

FIGURE 1: The UTO emergency alternative evaluation construction using the FAHP method.

Definition 2. Let $\alpha=\left\{\alpha_{1}, \alpha_{2}, \ldots, \alpha_{n}\right\}$ be a set of alternatives. Vectors $p=\left\{p_{1}, p_{2}, \ldots, p_{n}\right\}^{T}$ and $w=\left\{w_{1}, w_{2}, \ldots, w_{m}\right\}^{T}$ are two sets of weighting vectors, with $\sum_{i=0}^{n} p_{i}=1, p_{i} \in$ $[0,1], \sum_{i=0}^{m} w_{i}=1, w_{i} \in[0,1]$.

Definition 3. Let the vector $\left\{\alpha_{\sigma(1)}, \alpha_{\sigma(2)}, \ldots, \alpha_{\sigma(n)}\right\}$ be a permutation of vector $\alpha=\left\{\alpha_{1}, \alpha_{2}, \ldots, \alpha_{n}\right\}$, such that $\alpha_{\sigma(1)} \geq$ $\alpha_{\sigma(2)} \geq \cdots \geq \alpha_{\sigma(n)}$.

A WOWA operator of dimension $n$ is a mapping function: $R^{n} \rightarrow R$ with the two weighting vectors of $p=$ $\left\{p_{1}, p_{2}, \ldots, p_{n}\right\}^{T}$ and $w=\left\{w_{1}, w_{2}, \ldots, w_{m}\right\}^{T}$. The WOWA operator is defined as follows:

$$
\begin{aligned}
& \operatorname{WOWA}\left(\alpha_{1}, \alpha_{2}, \ldots, \alpha_{n}\right)=\sum_{i=0}^{n} v_{i} \alpha_{\sigma(i)} \\
& \text { with } v_{i}=\omega^{*}\left(\sum_{j \leq i} p_{\sigma(j)}\right)-\omega^{*}\left(\sum_{j<i} p_{\sigma(j)}\right),
\end{aligned}
$$

where $\omega^{*}$ is an increasing function interpolating points $\left(i / m, \sum_{j \leq i} w_{j}\right)$ together with the point $(0.0)$. Function $\omega^{*}$ is required to be a straight line when the points can be interpolated in this way $[29,36]$.

\section{Hybrid Method for Selection of Emergency Alternatives for UTO Metro System}

4.1. Defining the Preference with Linguistic Term. From the investigation of Line 10 in Shanghai, it can be concluded that the criteria used in UTO metro system are the safety of passengers $(\mathrm{C} 1)$, the emergency response time $(\mathrm{C} 2)$, and the performance of emergency recovery (C3). Applying the FAHP method to evaluate emergency alternatives according to emergency criteria is shown in Figure 1. However, the dispatchers cannot make accurate decision precisely with uncertain environment and usually have vague description to describe their preferences or judgments. To obtain the
TABLE 2: Linguistic terms for the comparison of emergency alternative evaluation and corresponding fuzzy preference values.

\begin{tabular}{lc}
\hline Linguistic terms & Fuzzy preference values \\
\hline Very good (VG) & 0.9 \\
Good (G) & 0.7 \\
Medium (M) & 0.5 \\
Bad (B) & 0.3 \\
Very bad (VB) & 0.1 \\
\hline
\end{tabular}

precise value, linguistic terms are taken by dispatchers to give their preferences in a familiar style. Table 2 gives the set of linguistic terms and their respective responding values. The preference value of pair to pair comparison to some alternatives can be derived according to the linguistic terms. For example, a traffic dispatcher would use the word "Good" rather than give the precise value difficultly when he makes decision with comparing two dispatching commands. Then the precise preference value 0.9 can be obtained from linguistic term in fuzzy preference value table.

As shown in Figure 1, it is supposed that there are $m$ dispatchers as decision makers $\left(D_{1}, D_{2}, \ldots, D_{m}\right)$ in a decision team in case of UTO metro emergency for evaluating $n$ alternatives $\left(\mathrm{EA}_{1}, \mathrm{EA}_{2}, \ldots, \mathrm{EA}_{n}\right)$ according to the three criteria $(\mathrm{C} 1, \mathrm{C} 2, \mathrm{C} 3)$. Fortunately, each dispatcher has the familiar and rational knowledge with the relative weights of the proposed three criteria because of the excellent safe culture of metro organization and normal training process for metro staff in China. So, we suppose the weights of the three criteria are $\{0.5,0.3,0.2\}$ in a unified pattern, based on interviews with dispatchers working in OCC. Each dispatcher $D_{k}$ has his (or her) relative evaluation $w_{i j}$ between alternative $\mathrm{EA}_{i}$ and alternative $\mathrm{EA}_{j}$ with respect to each criterion. Therefore, there will be a decision matrix $G\left[u\left(w_{i j}\right)_{n \times n}\right]_{m \times 3}$, where $u\left(w_{i j}\right)_{n \times n}$ is the pair to pair evaluation matrix among all emergency alternatives $n$. The exact value of $w_{i j}$ can be obtained from the linguistic terms in Table 2. 


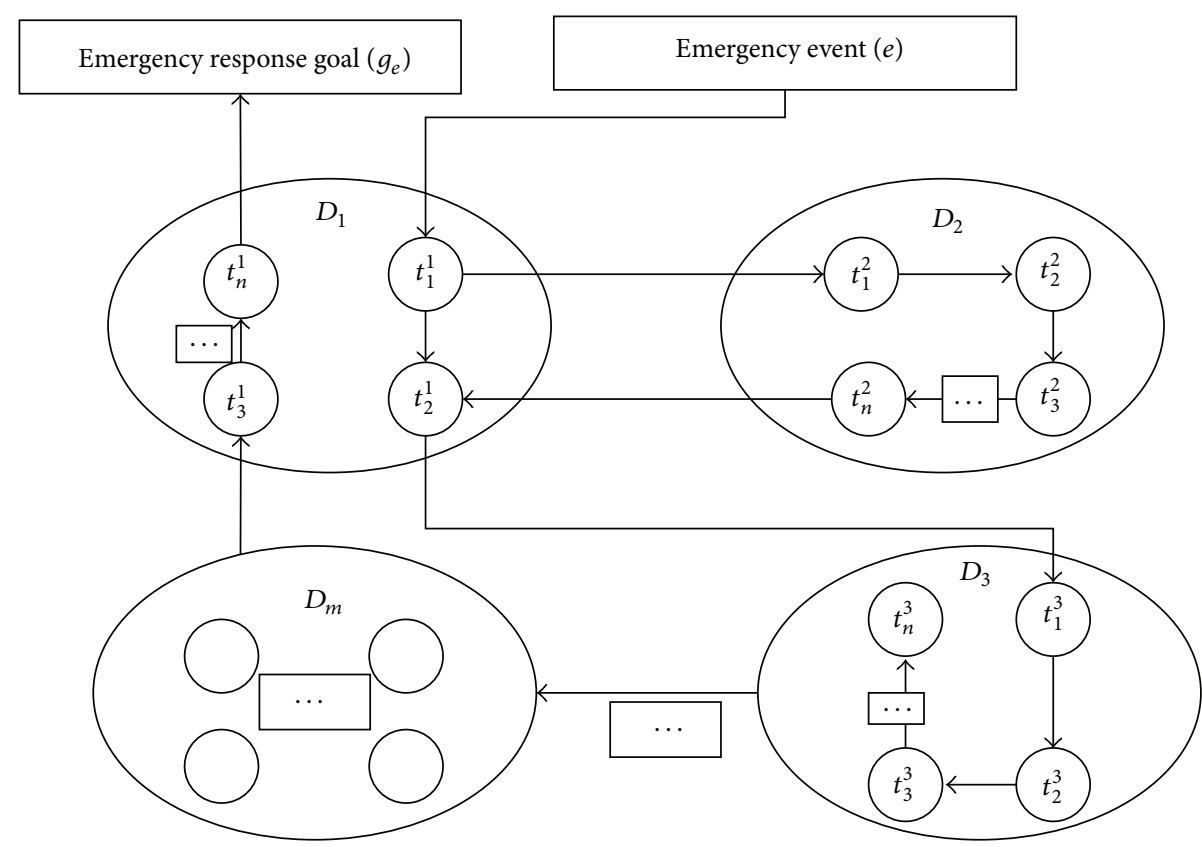

FIGURE 2: The emergency response task model of dispatchers in OCC.

\subsection{The Calculating Approach for Enhanced WOWA Operator Weights}

4.2.1. Determining the Importance Weights of Dispatcher In order to minimize the loss of emergency on operation services, the dispatchers try to carry out necessary and effective tasks to deal with the emergency and restore the disturbed metro system. For example, in case of a switch failure in front of the track, the traffic dispatcher should execute three tasks: (1) locking the failure zone to avoid the entrance of train, (2) setting the temporary train route to maintain continuous revenue service, and (3) adjusting the operation timetable to adapt to the failure event. The more the dispatcher participates in the emergency response process, the more important his/her duty is. From this point of view, the importance weights of dispatchers are dependent on their specific job responsibilities which are indicated by the emergency tasks number.

Moreover, each dispatcher works in his/her own domain and derives the necessary tasks at the operational level under current emergency to meet the arising requirements after the event. The operational steps are formed with the cooperation of different dispatchers. These dispatchers undertake tasks with different contents and numbers, which are relative to the type of emergency event. For example, in case of person falling onto the platform track, the traffic dispatcher confirms to brake the train, the power dispatcher cuts off the electricity of the third rail, and the passenger dispatcher provides the easing service to passengers in the train to prevent the second damage. It also implies that the dispatchers in OCC as decision maker have a close and entire dependence on the current emergency goal, and the goal of emergency response determines the contents of the tasks and the following operations. Therefore, the dependence of dispatcher related to the emergency goals under uncertain environment is another important aspect to define the importance of dispatchers.

The remote characteristic of management and supervision for the UTO metro system determinates that the performance of emergency response process under emergency highly depends on dispatchers and their tasks involved in the emergency event. The dispatchers in OCC should cooperate with each other according to the priorities of their task relations. Considering these factors, an OCC emergency response task model was proposed to cover three aspects: emergency goals, emergency related dispatcher, and dispatcher related tasks under each emergency event. It is noted that the key points of this study include the importance of decision makers and how their preference decisions of emergency alternatives are aggregated. Hence, we constructed the OCC emergency response task model to analyze the dependence relationship between different tasks and to obtain importance weights of dispatchers. There are five steps for construction of the emergency response task model of UTO metro system, as shown in Figure 2.

Step 1. Confirm the type and scope of emergency event $e$, represented by node of rectangle.

Step 2. Set the goal $g_{e}$ of emergency response, represented by node of rectangle.

Step 3. Identify the dispatchers related to the emergency, represented by node of ellipse, which may include $m$ dispatchers $\left\{D_{1}, D_{2}, \ldots, D_{m}\right\}$.

Step 4. Select the tasks following the dispatchers, represented by node of circle, which may include $n$ tasks $\left\{t_{1}^{k}, t_{2}^{k}, \ldots, t_{n}^{k}\right\}$ of dispatcher $k$ with $1 \leq k \leq m$. 
Step 5. For each task, add a directed edge to denote the sequence and priority between the tasks selected in Step 4. The last task will be executed at the end of task network and reach the emergency response goal $g_{e}$.

After the type and scope of emergency are identified from the remote alarming and failure information in Step 1, Step 2 defines emergency goals explicitly according to the urgency level and scope of emergency. Then, the imperative phase of emergency response process categorizes dispatchers involved in the emergency decision-making operation and their necessary tasks in Steps 3 to 5.

As aforementioned, the calculating formula of importance weight (IW) of dispatcher is proposed before finding out the appropriate priority of emergency decisions. The formulation refers to the typical proximity prestige defined by Wasserman and Faust [37] and takes into consideration the concept of decision maker involved in the task network.

The IW of dispatcher $k$ is defined by

$$
\mathrm{IW}_{k}=\sum_{i \in T^{k}} \frac{N_{1}^{k} / \sum_{k=1}^{m} N_{k}}{\sum_{j \in N_{1}^{k}}\left(d\left(t_{j}, t_{i}^{k}\right) / N_{1}^{k}\right)}
$$

As shown in (2), $M=\{1,2, \ldots, m \mid m \in N\}$ is assumed as the set of different types of dispatchers in OCC. The set of $T^{k}=\left\{t_{1}^{k}, t_{2}^{k}, \ldots, t_{n}^{k}\right\}$ is composed of dispatcher $k$ related tasks with respect to the emergency goals and the responsibility of each dispatcher. $N_{k}$ represents the number of tasks involved in dispatcher $D_{k}$. $N_{1}^{k}$ denotes the number of tasks outside dispatcher $D_{k}$ but it can reach to dispatcher $D_{k}$. $d\left(t_{j}, t_{i}^{k}\right)$ represents the distance of task $t_{j}$ outside dispatcher $D_{k}$ reachable to task $t_{i}^{k}$. The IW of dispatcher $k$ is the sum of the ratio of reachable tasks and average distance of all tasks reachable to dispatcher $D_{k}$.

From the emergency task network, we consider the $\mathrm{IW}_{k}$ as the ratio of the average reachable distance of all tasks related with the dispatcher $k$. According to (2), dispatcher $k$ becomes more important when (1) dispatcher $k$ should execute more tasks to reach $g^{e}$; (2) more tasks executed by other dispatchers should be cooperated with dispatch $k$; (3) the distance between the tasks $t_{i}^{k}$ and $t_{j}$ is shorter. The normalization process is necessary to obtain the sum of $\mathrm{IW}_{k}^{\prime}$ equal to 1 , as shown in the following:

$$
\mathrm{IW}_{k}^{\prime}=\frac{\mathrm{IW}_{k}}{\sum_{k=1}^{m} \mathrm{IW}_{k}} \text {. }
$$

4.2.2. Determining the OWA Operator Weights of Dispatcher. The OWA operator uses different decision criteria such as Maximax (optimistic) and Maximin (pessimistic), to express the optimism degree of the decision maker [38]. The OWA operator weights are measured by two important parameters: dispersion (or entropy) and orness, which indicate the entropy of the probability distribution and the measure of the optimism of the decision maker, respectively [26]. Fuller and Majlender [39] introduced a method of minimizing the variance of OWA operator weights under a given level of orness. Wang and Parkan [40] employed a linear programming (LP) to minimize the maximum disparity between two adjacent weights under a given level of orness. Wang et al. [41] presented OWA operator weights named least squares deviation (LSD), minimizing the sum square of deviation between $w_{i}$ and $w_{i+1}$. In this paper, fuzzy membership function was employed to obtain the $n$ dimensional OWA operator weights [42].

The OWA weights vector $w_{q}^{*}$ is calculated by [42]

$$
\begin{array}{r}
w_{q}^{*}=Q\left(\frac{q}{n}\right)-Q\left(\frac{(q-1)}{n}\right) \\
\text { with } w_{q}^{*} \geq 0, \sum_{q=1}^{n} w_{q}^{*}=1,
\end{array}
$$

where $Q$ is a fuzzy membership function denoted by

$$
Q(r)= \begin{cases}0, & r<\alpha, \\ \frac{r-\alpha}{\beta-\alpha}, & \alpha \leq r \leq \beta, \\ 1, & r>\beta,\end{cases}
$$

with parameter $(\alpha, \beta)=(0.3,0.8)$, indicating the fuzzy principle of majority.

\subsubsection{Determining the Enhanced WOWA Operator Weights.} The importance weights could be used to evaluate the prominence of dispatcher as decision maker based on the proposed emergency response task model. However, the model is insufficient to represent the logic of the emergency decision selection with one single factor of the importance weight of dispatcher because different decision makers have different preferences based on their knowledge and experience. Therefore, it is necessary to aggregate the preferences of group decision regarding the optimism degree and prominence of decision makers.

Adapting to the characteristics of the UTO metro system, an approach of the enhanced WOWA operator is proposed to assign the importance of the dispatchers to the ordered weights, solving emergency alternatives selection from multidispatcher of UTO metro. In this systematic approach, relationship between dispatchers and dispatcher related tasks under the emergency goal is modelled to derive the importance weights, and a fuzzy membership function is integrated to decide the OWA operator weights. There are four steps for this approach. Suppose there are $m$ dispatchers $\{1,2, \ldots, m\}$ in OCC.

Step 1. Construct the emergency response task model proposed in Section 4.2.1.

Step 2. Let $w_{k}=\left\{w_{1}, w_{2}, \ldots, w_{m}\right\}^{T}$ be the importance weights of dispatchers. The weights vector is calculated by (2) and (3).

Step 3. Let $w_{q}^{*}=\left\{w_{1}^{*}, w_{2}^{*}, \ldots, w_{m}^{*}\right\}^{T}$ be the OWA weights of preference relationship. The weights vector is calculated by (4) and (5). 


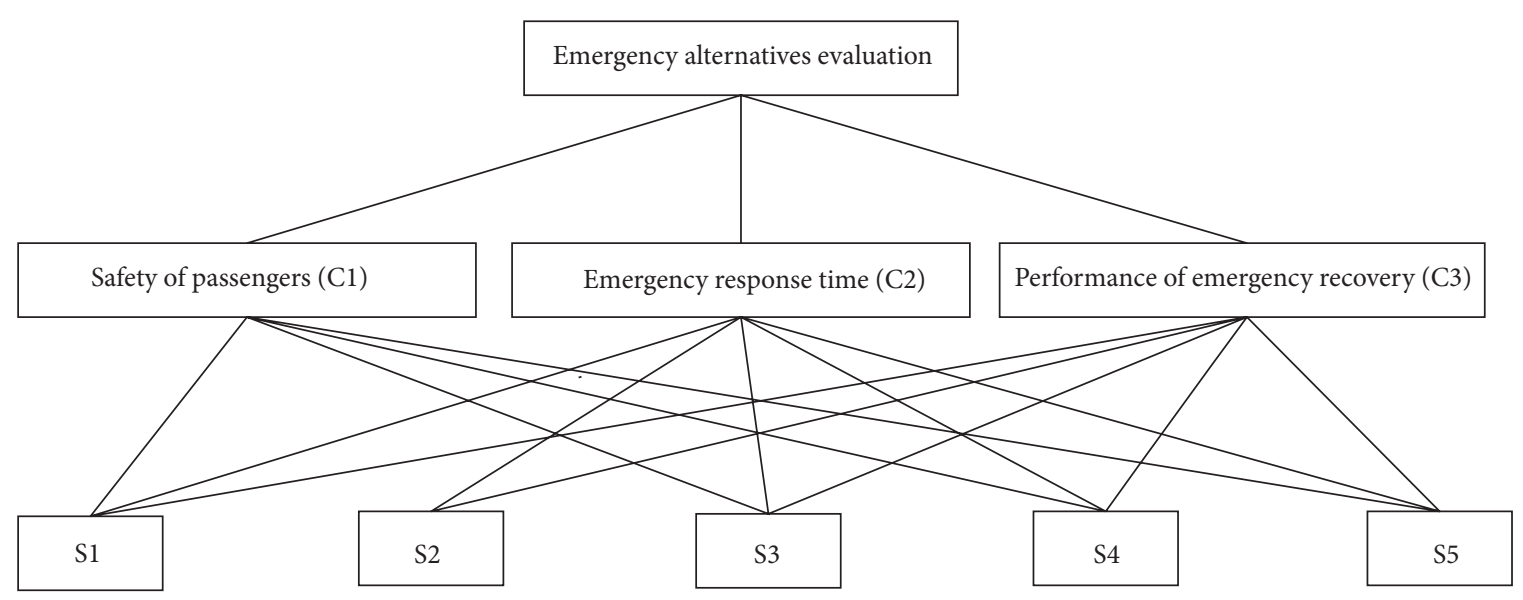

FIGURE 3: The AHP emergency alternative evaluation model in case of a train fire.

Step 4. The aggregation weights vector $v=\left\{v_{1}, v_{2}, \ldots, v_{m}\right\}^{T}$ is calculated by (1) based on the importance weights of dispatchers and OWA weights.

4.3. Integrating the Enhanced WOWA Operator with FAHP Method. The approach, integrating the proposed enhanced WOWA operator with FAHP method to obtain the ultimate priority, is described as follows.

Step 1. $D=\left\{D_{1}, D_{2}, \ldots, D_{m}\right\}$ is assumed as a set of dispatchers and $P=\left\{P^{(1)(s)}, P^{(2)(s)}, \ldots, P^{(m)(s)}\right\}$ is assumed as the set of preference judgment vectors under criterion $s$, where $P^{(k)(s)}=u\left(w_{i j}{ }^{k(s)}\right)_{n \times n}$. The aggregation matrix $p_{i j}^{* s}$ of preference judgment under criterion $s$ is formulated with enhanced WOWA operator by

$$
p_{i j}^{* s}=\sum_{l=0}^{m} v_{l} p_{l}
$$

where $p_{l}$ is $l$ th largest value of $w_{i j}{ }^{k(s)}$ with $1 \leq k \leq m$.

Step 2. The aggregation matrix of multidispatcher with respect to criterion $s$ is $p^{* s}=\left(p_{i j}^{* s}\right)_{n \times n}, 1 \leq s \leq 3$. The dispatchers in UTO metro have the same perception of the weights of the criteria, so the multicriteria aggregation matrix $p_{i j}^{\tau}$ can be calculated by

$$
p_{i j}^{\tau}=\sum_{s=0}^{3} \lambda_{s} p_{i j}^{* s},
$$

where $\lambda=\left(\lambda_{1}, \lambda_{2}, \lambda_{3}\right)=(0.5,0.3,0.2)$ is the weights of criteria, and (7) uses the weighted average method to derive the ultimate matrix.

Step 3. The degree indicator $r_{i}$ demonstrates that alternative $i$ is superior to other alternatives and, from the fuzzy principle of majority, it is calculated by

$$
r_{i}=\theta\left(p_{i j}^{\tau}, j=1,2, \ldots, n\right)=\sum_{q=1}^{n} v_{q} c_{i}^{q}, \quad i \in m,
$$

where $v_{q}$ is obtained from (1) with parameter $(\alpha, \beta)=$ $(0.3,0.8)$, and $c_{i}^{q}$ is $q$ th largest element of set $\left\{p_{i j}^{\tau} \mid j=\right.$ $1,2, \ldots, n\}$.

Step 4. The degree indicator $r_{i}^{\prime}$ is normalized by

$$
r_{i}^{\prime}=\frac{r_{i}}{\sum_{i=1}^{n} r_{i}}
$$

The priority of emergency alternatives is given from the vector $r^{\prime}=\left\{r_{1}^{\prime}, r_{2}^{\prime}, \ldots, r_{n}^{\prime}\right\}$.

\section{Case Study}

The proposed FAHP-WOWA method can be applied on decision support in an emergency event. It integrates all the opinions of dispatchers, and the relationships between the preferences and the importance of dispatchers in emergency response task situation are taken into consideration and combined properly. Here a train fire emergency during the operation process of the train between the stations is taken as a case.

In case of fire detection, the aforementioned three criteria in the metro emergency are employed to construct the AHP model shown in Figure 3, in which there are five emergency alternatives, represented by $\mathrm{S} 1$ to $\mathrm{S} 5$, respectively, which are explained in Table 3.

It is assumed that $D=\{$ Traffic dispatcher, Environment dispatcher, Passenger Dispatcher, Vehicle Dispatcher, Power dispatcher\} is the set of decision makers in OCC.

5.1. Calculating Enhanced WOWA Operator Weights. After the analysis of FAHP applied on aforementioned train fire case, the enhanced WOWA operator weights are calculated as follows.

Step 1. The emergency response task model is constructed as shown in Figure 4. The detailed tasks according to the responsibilities of the dispatchers are summarized in Table 4. 
TABLE 3: Emergency alternatives of train fire.

\begin{tabular}{ll}
\hline Number & Alternatives \\
S1 & $\begin{array}{l}\text { Allowing the train to continue its ride to the next safe place (e.g., next station) where the train can be stopped } \\
\text { and immobilized. } \\
\text { Holding the train between stations, and the train door is manually opened by passengers under the guidance of } \\
\text { S2 }\end{array}$ \\
S3 broadcast from OCC. \\
The passengers will be evacuated by the work staff between the stations. \\
S5 & The passengers will be evacuated under the guidance of the broadcast from OCC between the stations. \\
\end{tabular}

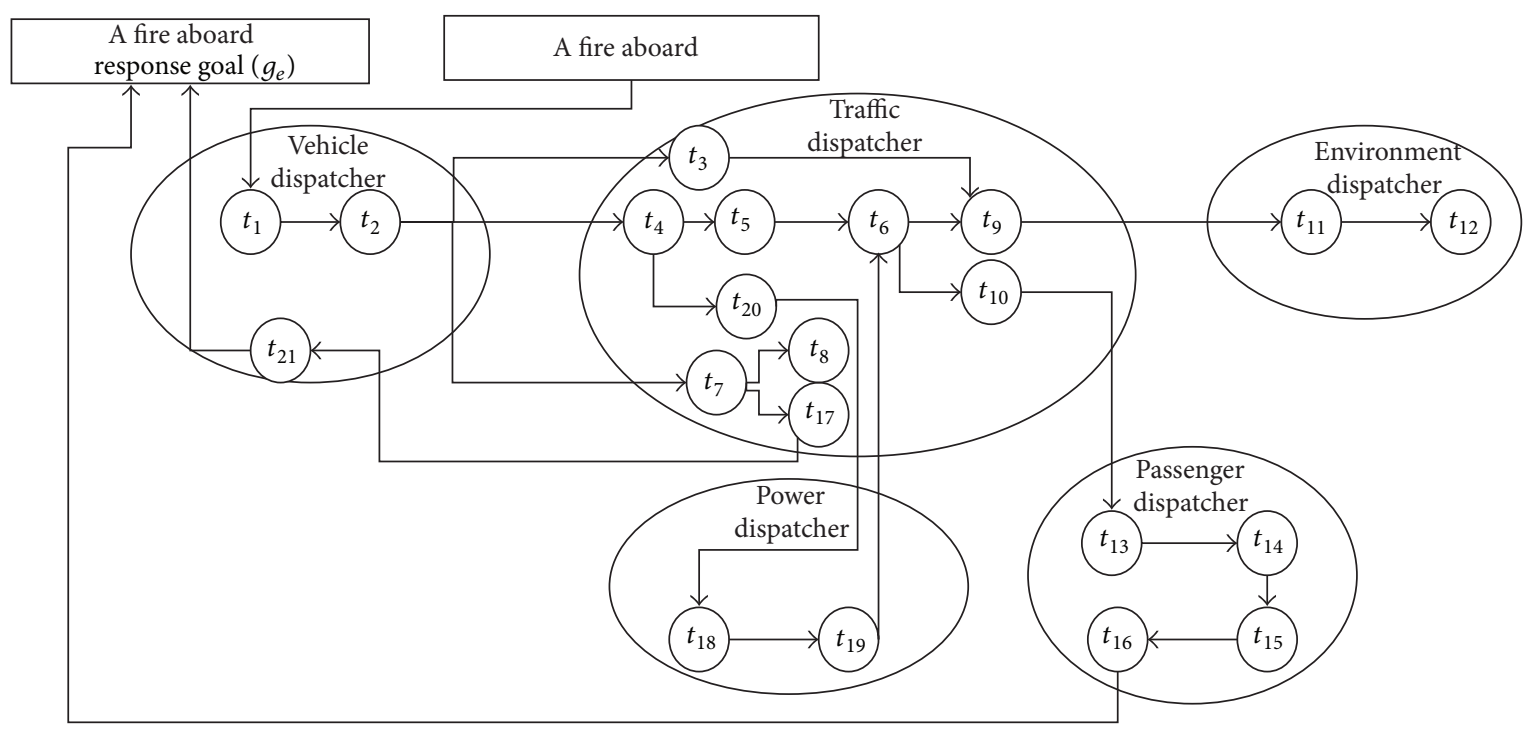

FIGURE 4: Emergency response task model in case of train fire.

Step 2. Based on the emergency response task model in case of train fire, the importance weights vector of dispatchers $w_{k}$ defined in set $D$ is calculated by (2) and (3): $w_{k}=$ $\{0.53,0.07,0.11,0.16,0.13\}^{T}$.

Step 3. The preference matrixes of different dispatchers under the criterion of safety of passengers are presented from Tables 5-9 based on the interview of dispatchers in OCC.

The OWA operator weights vector of dispatcher $w_{q}^{*}$ is obtained by (4) and (5): $w_{q}^{*}=\{0,0.2,0.4,0.4,0\}^{T}$.

Step 4. Based on the vectors $w_{k}$ and $w_{q}^{*}$, the aggregation weights vector can be calculated by (1): $v=$ $\{0.6,0.27,0.13,0,0\}^{T}$.

5.2. Aggregating the Preferences of Dispatchers with WOWA Operator and FAHP. The final priority of emergency alternatives is obtained through the aggregation process of fuzzy preferences derived from the linguistic terms based on FAHP method and enhanced WOWA operator weights. The process is described as follows.

Step 1. From the approach discussed in Section 4.3, the preference aggregation matrixes of different dispatchers under multicriteria are calculated by (6) and they are shown from Tables 10-12.

Step 2. The aggregation matrix of multidispatcher is obtained by (7) and it is shown in Table 13.

Step 3. Degree indicators vector $r$, which demonstrates that one alternative is superior to other alternatives, is calculated by (8):

$$
r=\{0.8989,0.7988,0.4394,0.5631,0.6929\}^{T} .
$$

Step 4. After normalization with (9), the priority weights vector $r^{\prime}$ is

$$
r^{\prime}=\{0.2649,0.2354,0.1295,0.1659,0.2042\}^{T},
$$

which means the sequence of the priority of emergency alternatives is

$$
\mathrm{S} 1>\mathrm{S} 2>\mathrm{S} 5>\mathrm{S} 4>\mathrm{S} 3 \mathrm{.}
$$

From the investigation on Line 10 in Shanghai, it is noted that the dispatchers always have the guideline in any emergency situations: dispatching the emergency train into station if the train can move. For this reason, the emergency alternative S1, allowing the train to continue running to next 
TABLE 4: Detailed tasks in case of train fire.

\begin{tabular}{ll}
\hline Task sequence & Task content \\
\hline$t_{1}$ & Monitoring fire alarm indicator \\
$t_{2}$ & Communicating with traffic dispatcher \\
$t_{3}$ & Holding train at next station \\
$t_{4}$ & Opening platform doors \\
$t_{5}$ & Molding train between stations \\
$t_{6}$ & Communicating with passenger dispatcher \\
$t_{7}$ & Communicating with environment dispatcher \\
$t_{8}$ & Communicating with station work staff \\
$t_{9}$ & Establishing zone of protection \\
$t_{10}$ & Communicating with fire departments \\
$t_{11}$ & Releasing fire on board emergency plan \\
$t_{12}$ & Monitoring coach \\
$t_{13}$ & Communicating with passengers \\
$t_{14}$ & Releasing passenger evacuation information \\
$t_{15}$ & Monitoring passenger evacuation process \\
$t_{16}$ & Communicating with vehicle dispatcher \\
$t_{17}$ & Cutting off third rail power \\
$t_{18}$ & Communicating with traffic dispatcher \\
$t_{19}$ & Communicating with power dispatcher \\
$t_{20}$ & Opening train doors \\
$t_{21}$ &
\end{tabular}

TABLE 5: Preference matrix of traffic dispatcher.

\begin{tabular}{lccccc}
\hline $\begin{array}{l}\text { Emergency } \\
\text { alternatives }\end{array}$ & S1 & S2 & S3 & S4 & S5 \\
\hline S1 & M & VG & VG & VG & G \\
S2 & VB & M & VG & G & B \\
S3 & VB & VB & M & G & VB \\
S4 & VB & B & B & M & B \\
S5 & B & G & VG & G & M \\
\hline
\end{tabular}

TABLE 6: Preference matrix of environment dispatcher.

\begin{tabular}{lccccc}
\hline $\begin{array}{l}\text { Emergency } \\
\text { alternatives }\end{array}$ & S1 & S2 & S3 & S4 & S5 \\
\hline S1 & M & VG & VG & VG & G \\
S2 & VB & M & VG & G & B \\
S3 & VB & VB & M & G & VB \\
S4 & VB & B & B & M & B \\
S5 & B & G & VG & G & M \\
\hline
\end{tabular}

safe place, is the first selection. The alternative of holding the train between stations (S2) is the second selection. After making the emergency decision of the fired train, the alternatives of evacuating passengers should have a proper priority decision, and S5, evacuating by the station staff on the platform, is the third selection for the aforementioned guideline. The alternative S4 of evacuating passengers by the broadcast from OCC between the stations is the fourth selection for its inflexibility and uncontrollability. Then the alternative S3 of evacuating the passengers by the work staff
TABLE 7: Preference matrix of passenger dispatcher.

\begin{tabular}{lccccc}
\hline $\begin{array}{l}\text { Emergency } \\
\text { alternatives }\end{array}$ & S1 & S2 & S3 & S4 & S5 \\
\hline S1 & M & B & B & B & G \\
S2 & G & M & G & VG & VG \\
S3 & G & B & M & G & VG \\
S4 & G & VB & B & M & VG \\
S5 & B & VB & VB & VB & M \\
\hline
\end{tabular}

TABLE 8: Preference matrix of vehicle dispatcher.

\begin{tabular}{lccccc}
\hline $\begin{array}{l}\text { Emergency } \\
\text { alternatives }\end{array}$ & S1 & S2 & S3 & S4 & S5 \\
\hline S1 & M & VG & VG & VG & VG \\
S2 & VB & M & G & VG & G \\
S3 & VB & B & M & G & B \\
S4 & VB & VB & B & M & VB \\
S5 & VB & B & G & VG & M \\
\hline
\end{tabular}

TABle 9: Preference matrix of power dispatcher.

\begin{tabular}{lccccc}
\hline $\begin{array}{l}\text { Emergency } \\
\text { alternatives }\end{array}$ & S1 & S2 & S3 & S4 & S5 \\
\hline S1 & M & VG & VG & VG & G \\
S2 & VB & M & G & VG & B \\
S3 & VB & B & M & G & B \\
S4 & VB & VB & B & M & VB \\
S5 & B & G & G & VG & M \\
\hline
\end{tabular}

TABLE 10: Aggregation matrix under criterion of the safety of passengers $(\mathrm{Cl})$.

\begin{tabular}{lccccc}
\hline $\begin{array}{l}\text { Emergency } \\
\text { alternatives }\end{array}$ & S1 & S2 & S3 & S4 & S5 \\
\hline S1 & 0.5000 & 0.9000 & 0.9000 & 0.9000 & 0.5572 \\
S2 & 0.6340 & 0.5000 & 0.8740 & 0.9000 & 0.7680 \\
S3 & 0.4600 & 0.3000 & 0.5000 & 0.5400 & 0.2200 \\
S4 & 0.4600 & 0.1811 & 0.6480 & 0.5000 & 0.6600 \\
S5 & 0.3000 & 0.5140 & 0.6220 & 0.6220 & 0.5000 \\
\hline
\end{tabular}

TABLE 11: Aggregation matrix under criterion of the emergency response time (C2).

\begin{tabular}{lccccc}
\hline $\begin{array}{l}\text { Emergency } \\
\text { alternatives }\end{array}$ & S1 & S2 & S3 & S4 & S5 \\
\hline S1 & 0.5000 & 0.8740 & 0.9000 & 0.9000 & 0.5572 \\
S2 & 0.8220 & 0.5000 & 0.8200 & 0.7000 & 0.5400 \\
S3 & 0.6220 & 0.1000 & 0.5000 & 0.3000 & 0.2740 \\
S4 & 0.6220 & 0.1832 & 0.7000 & 0.5000 & 0.8220 \\
S5 & 0.3000 & 0.6480 & 0.8200 & 0.8740 & 0.5000 \\
\hline
\end{tabular}

between the stations is the last selection. The weakness of this alternative is that the rescue time will be lost if station staff 
TABLE 12: Aggregation matrix under criterion of the performance of emergency recovery $(\mathrm{C} 3)$.

\begin{tabular}{lccccc}
\hline \multirow{2}{*}{$\begin{array}{l}\text { Emergency } \\
\text { alternatives }\end{array}$} & \multicolumn{5}{c}{ C3: the performance of emergency recovery } \\
& S1 & S2 & S3 & S4 & S5 \\
\hline S1 & 0.5000 & 0.9000 & 0.9000 & 0.9000 & 0.4372 \\
S2 & 0.5800 & 0.5000 & 0.9000 & 0.7000 & 0.6600 \\
S3 & 0.4600 & 0.1000 & 0.5000 & 0.3000 & 0.5800 \\
S4 & 0.4600 & 0.1832 & 0.7000 & 0.5000 & 0.6600 \\
S5 & 0.3000 & 0.6220 & 0.7960 & 0.6220 & 0.5000 \\
\hline
\end{tabular}

TABLE 13: Aggregation matrix under multicriteria.

\begin{tabular}{lccccc}
\hline $\begin{array}{l}\text { Emergency } \\
\text { alternatives }\end{array}$ & S1 & S2 & S3 & S4 & S5 \\
\hline S1 & 0.5000 & 0.8922 & 0.9000 & 0.9000 & 0.5332 \\
S2 & 0.6796 & 0.5000 & 0.8630 & 0.8000 & 0.6780 \\
S3 & 0.5086 & 0.2000 & 0.5000 & 0.4200 & 0.3082 \\
S4 & 0.5086 & 0.1821 & 0.6740 & 0.5000 & 0.7086 \\
S5 & 0.3000 & 0.5758 & 0.7162 & 0.6976 & 0.5000 \\
\hline
\end{tabular}

goes into the guideway between stations. Also the fire in the train may cause a second damage to passengers.

In summary, the result of the aggregation method of dispatcher's preferences applied on the case of a train fire during the operation process between the stations is consistency with the real emergency guidance in the situation of UTO system.

\section{Conclusion}

The thinking and preference of dispatcher group in OCC have a key impact on the performance of emergency response for their emergency responding behaviors. To find out the optimized emergency solution, a hybrid decision-making method is proposed to solve group decision-making support problem of emergency alternatives in UTO metro system of China.

The linguistic terms adopted in FAHP avoid the vagueness of dispatchers and have a responding relation with fuzzy preference to get precise value. For ranking purpose, it is important to choose an appropriate WOWA operator weight applied on UTO metro system to aggregate the preferences of dispatcher group. The interrelationship between the dispatchers and their respective preferences is calculated through assigning the dispatcher importance to OWA operator weights. When evaluating the importance of dispatcher in OCC, the proposed formulation process considers task interdependencies featured by the structure of the corresponding emergency task model. Through integrating the FAHP method with the enhanced WOWA, the priority of emergency alternatives is obtained to support the complex emergency response procedure in new UTO system. This proposed method has been demonstrated and partially validated through a case study of train fire. In this way, the dispatcher group facing UTO emergency event would focus on performance of emergency response procedure rather
TABLE 14: Preference matrix of traffic dispatcher.

\begin{tabular}{lccccc}
\hline Emergency alternatives & S1 & S2 & S3 & S4 & S5 \\
\hline S1 & M & VG & VG & G & G \\
S2 & VB & M & VG & G & VG \\
S3 & VB & VB & M & B & VG \\
S4 & B & B & G & M & VG \\
S5 & B & VB & VB & VB & M \\
\hline
\end{tabular}

TABle 15: Preference matrix of environment dispatcher.

\begin{tabular}{lccccc}
\hline Emergency alternatives & S1 & S2 & S3 & S4 & S5 \\
\hline S1 & M & VG & VG & VG & G \\
S2 & VB & M & VG & G & B \\
S3 & VB & VB & M & B & VB \\
S4 & VB & B & G & M & B \\
S5 & B & G & VG & G & M \\
\hline
\end{tabular}

TABLE 16: Preference matrix of passenger dispatcher.

\begin{tabular}{lccccc}
\hline Emergency alternatives & S1 & S2 & S3 & S4 & S5 \\
\hline S1 & M & G & G & G & G \\
S2 & B & M & G & G & B \\
S3 & B & VB & M & B & VG \\
S4 & B & B & G & M & VG \\
S5 & B & G & VB & VB & M \\
\hline
\end{tabular}

TABLe 17: Preference matrix of vehicle dispatcher.

\begin{tabular}{lccccc}
\hline Emergency alternatives & S1 & S2 & S3 & S4 & S5 \\
\hline S1 & M & G & VG & VG & VG \\
S2 & B & M & G & VG & G \\
S3 & VB & B & M & G & B \\
S4 & VB & VB & B & M & VB \\
S5 & VB & B & G & VG & M \\
\hline
\end{tabular}

TABLE 18: Preference matrix of power dispatcher.

\begin{tabular}{lccccc}
\hline Emergency alternatives & S1 & S2 & S3 & S4 & S5 \\
\hline S1 & M & VG & VG & VG & G \\
S2 & VB & M & G & VG & B \\
S3 & VB & B & M & G & B \\
S4 & VB & VB & B & M & VB \\
S5 & B & G & G & VG & M \\
\hline
\end{tabular}

than be confused by excessive decision information under multiple criteria and multiple decision makers.

Further study will focus on aggregation method of incomplete information of dispatcher's preference and validate the algorithm on the agent based pedestrian simulation model in several emergency scenarios.

\section{Appendix}

The preference matrixes of different dispatchers under the criterion of emergency response time are present in Tables 14-18. 
TABLE 19: Preference matrix of traffic dispatcher.

\begin{tabular}{lccccc}
\hline Emergency alternatives & S1 & S2 & S3 & S4 & S5 \\
\hline S1 & M & VG & VG & VG & G \\
S2 & VB & M & VG & G & B \\
S3 & VB & VB & M & B & VB \\
S4 & VB & B & G & M & B \\
S5 & B & G & VG & G & M \\
\hline
\end{tabular}

TABle 20: Preference matrix of environment dispatcher.

\begin{tabular}{lccccc}
\hline Emergency alternatives & S1 & S2 & S3 & S4 & S5 \\
\hline S1 & M & VG & VG & VG & G \\
S2 & VB & M & VG & G & B \\
S3 & VB & VB & M & B & VB \\
S4 & VB & B & G & M & B \\
S5 & B & G & VG & G & M \\
\hline
\end{tabular}

TABLE 21: Preference matrix of passenger dispatcher.

\begin{tabular}{lccccc}
\hline Emergency alternatives & S1 & S2 & S3 & S4 & S5 \\
\hline S1 & M & VB & B & B & G \\
S2 & VG & M & VG & G & VG \\
S3 & G & VB & M & B & VG \\
S4 & G & B & G & M & VG \\
S5 & B & VB & VB & VB & M \\
\hline
\end{tabular}

TABle 22: Preference matrix of vehicle dispatcher.

\begin{tabular}{lccccc}
\hline Emergency alternatives & S1 & S2 & S3 & S4 & S5 \\
\hline S1 & M & VG & VG & VG & G \\
S2 & VB & M & VG & G & B \\
S3 & VB & VB & M & B & VB \\
S4 & VB & B & G & M & B \\
S5 & B & G & VG & G & M \\
\hline
\end{tabular}

TABle 23: Preference matrix of power dispatcher.

\begin{tabular}{lccccc}
\hline Emergency alternatives & $\mathrm{M}$ & $\mathrm{VG}$ & $\mathrm{VG}$ & $\mathrm{VG}$ & $\mathrm{G}$ \\
\hline S1 & $\mathrm{VB}$ & $\mathrm{M}$ & $\mathrm{VG}$ & $\mathrm{G}$ & $\mathrm{B}$ \\
S2 & $\mathrm{VB}$ & $\mathrm{VB}$ & $\mathrm{M}$ & $\mathrm{B}$ & $\mathrm{VB}$ \\
S3 & $\mathrm{VB}$ & $\mathrm{B}$ & $\mathrm{G}$ & $\mathrm{M}$ & $\mathrm{B}$ \\
S4 & $\mathrm{B}$ & $\mathrm{G}$ & $\mathrm{VG}$ & $\mathrm{G}$ & $\mathrm{M}$ \\
S5 & $\mathrm{M}$ & $\mathrm{VG}$ & $\mathrm{VG}$ & $\mathrm{VG}$ & $\mathrm{G}$ \\
\hline
\end{tabular}

The preference matrixes of different dispatchers under the criterion of performance of emergency recovery are present in Tables 19-23.

\section{Conflict of Interests}

The authors declare that there is no conflict of interests regarding the publication of this paper.

\section{Acknowledgments}

This work was partly supported by Beijing Laboratory of Urban Rail Transit, Beijing Key Laboratory of Urban Rail Transit Automation and Control, Beijing Municipal Science and Technology Project (Project no. D141100000814002), the Fundamental Research Funds for the Central Universities (2015JBJ003), and the NSFC under U1434209.

\section{References}

[1] UITP, Press Kit Metro Automation Facts, Figures and TrendsA Global Bid for Automation: UITP Observatory of Automated Metros Confirms Sustained Growth Rates for the Coming Years, UITP, 2011, http://www.uitp.org/sites/default/files/ Metro\%20automation\%20-\%20facts\%20and\%20figures.pdff.

[2] W. Raskob and J. Ehrhardt, The RODOS System: Decision Support for Nuclear Off-Site Emergency Management in Europe, Forschungszentrum Karlsruhe, 2000.

[3] K. N. Papamichail and S. French, "Decision support in nuclear emergencies," Journal of Hazardous Materials, vol. 71, no. 1-3, pp. 321-342, 2000.

[4] J. Ehrhardt, J. Päsler-Sauer, O. Schüle, G. Benz, M. Rafat, and J. Richter, "Development of RODOS*, a comprehensive decision support system for nuclear emergencies in European overview," Radiation Protection Dosimetry, vol. 50, no. 2-4, pp. 195-203, 1993.

[5] J. Geldermann, V. Bertsch, M. Treitz, S. French, K. N. Papamichail, and R. P. Hämäläinen, "Multi-criteria decision support and evaluation of strategies for nuclear remediation management," Omega, vol. 37, no. 1, pp. 238-251, 2009.

[6] V. Bertsch, M. Treitz, J. Geldermann, and O. Rentz, "Sensitivity analyses in multi-attribute decision support for off-site nuclear emergency and recovery management," International Journal of Energy Sector Management, vol. 1, no. 4, pp. 342-365, 2007.

[7] K. G. Zografos, G. M. Vasilakis, and I. M. Giannouli, "Methodological framework for developing decision support systems (DSS) for hazardous materials emergency response operations," Journal of Hazardous Materials, vol. 71, no. 1, pp. 503-521, 2000.

[8] K. G. Zografos and K. N. Androutsopoulos, "A decision support system for integrated hazardous materials routing and emergency response decisions," Transportation Research Part C: Emerging Technologies, vol. 16, no. 6, pp. 684-703, 2008.

[9] M. Abrahamsson, H. Hassel, and H. Tehler, "Towards a systemoriented framework for analysing and evaluating emergency response," Journal of Contingencies and Crisis Management, vol. 18 , no. 1, pp. 14-25, 2010.

[10] D. Wang, C. Qi, and H. Wang, "Improving emergency response collaboration and resource allocation by task network mapping and analysis," Safety Science, vol. 70, pp. 9-18, 2014.

[11] J. Wang and W. Fang, "A structured method for the traffic dispatcher error behavior analysis in metro accident investigation," Safety Science, vol. 70, pp. 339-347, 2014.

[12] H. Karvonen, I. Aaltonen, M. Wahlström, L. Salo, P. Savioja, and L. Norros, "Hidden roles of the train driver: a challenge for metro automation," Interacting with Computers, vol. 23, no. 4, pp. 289-298, 2011.

[13] IEC, "Railway applications-urban guided transport management and command/control systems-part 2: functional requirements specification," Draft EN 62290-2, 2011, http:// webstore.iec.ch/Webstore/webstore.nsf/Artnum_PK/49864. 
[14] M. K. Lindell, C. Prater, and R. W. Perry, Wiley Pathways Introduction to Emergency Management, John Wiley \& Sons, 2006.

[15] Y. Ju and A. Wang, "Emergency alternative evaluation under group decision makers: amethod of incorporating DS/AHP with extended TOPSIS," Expert Systems with Applications, vol. 39, no. 1, pp. 1315-1323, 2012.

[16] D. Meng and Z. Pei, "On weighted unbalanced linguistic aggregation operators in group decision making," Information Sciences, vol. 223, pp. 31-41, 2013.

[17] T. L. Saaty, The Analytic Hierarchy Process, McGraw-Hill, New York, NY, USA, 1980.

[18] N. Subramanian and R. Ramanathan, "A review of applications of Analytic Hierarchy Process in operations management," International Journal of Production Economics, vol. 138, no. 2, pp. 215-241, 2012.

[19] H. Deng, "Multicriteria analysis with fuzzy pairwise comparison," International Journal of Approximate Reasoning, vol. 21, no. 3, pp. 215-231, 1999.

[20] T. Tanino, "Fuzzy preference orderings in group decision making," Fuzzy Sets and Systems, vol. 12, no. 2, pp. 117-131, 1984.

[21] J. Rezaei and R. Ortt, "Multi-criteria supplier segmentation using a fuzzy preference relations based AHP," European Journal of Operational Research, vol. 225, no. 1, pp. 75-84, 2013.

[22] C. V. Altrock and B. Krause, "Multi-criteria decision making in German automotive industry using fuzzy logic," Fuzzy Sets and Systems, vol. 63, no. 3, pp. 375-380, 1994.

[23] P.-L. Chang and Y.-C. Chen, "A fuzzy multi-criteria decision making method for technology transfer strategy selection in biotechnology," Fuzzy Sets and Systems, vol. 63, no. 2, pp. 131139, 1994.

[24] G.-H. Tzeng, T.-A. Shiau, and J.-Y. Teng, "Multiobjective decision-making approach to energy supply mix decisions in Taiwan," Energy Sources, vol. 16, no. 3, pp. 301-316, 1994.

[25] M. T. Tang and G. H. Tzeng, "A hierarchy fuzzy MCDM method for studying electronic marketing strategies in the information service industry," Journal of International Information Management, vol. 8, no. 1, pp. 1-22, 1998.

[26] R. R. Yager, “On ordered weighted averaging aggregation operators in multicriteria decisionmaking," IEEE Transactions on Systems, Man, and Cybernetics, vol. 18, no. 1, pp. 183-190, 1988.

[27] M. Grabisch, S. A. Orlovski, and R. R. Yager, "Fuzzy aggregation of numerical preferences," in Fuzzy Sets in Decision Analysis, Operations Research and Statistics, pp. 31-68, Springer US, 1988.

[28] G. R. Amin, "Notes on properties of the OWA weights determination model," Computers \& Industrial Engineering, vol. 52, no. 4, pp. 533-538, 2007.

[29] V. Torra, "The weighted OWA operator," International Journal of Intelligent Systems, vol. 12, no. 2, pp. 153-166, 1997.

[30] A. Valls and V. Torra, "Using classification as an aggregation tool in MCDM," Fuzzy Sets and Systems, vol. 115, no. 1, pp. 159-168, 2000.

[31] D. Nettleton and J. Muñiz, "Processing and representation of meta-data for sleep apnea diagnosis with an artificial intelligence approach," International Journal of Medical Informatics, vol. 63, no. 1-2, pp. 77-89, 2001.

[32] C.-S. Yu, "A GP-AHP method for solving group decisionmaking fuzzy AHP problems," Computers \& Operations Research, vol. 29, no. 14, pp. 1969-2001, 2002.
[33] Y. Ju, A. Wang, and X. Liu, "Evaluating emergency response capacity by fuzzy AHP and 2-tuple fuzzy linguistic approach," Expert Systems with Applications, vol. 39, no. 8, pp. 6972-6981, 2012.

[34] R. A. Ribeiro, "Fuzzy multiple attribute decision making: a review and new preference elicitation techniques," Fuzzy Sets and Systems, vol. 78, no. 2, pp. 155-181, 1996.

[35] R. R. Levary and K. Wan, "A simulation approach for handling uncertainty in the analytic hierarchy process," European Journal of Operational Research, vol. 106, no. 1, pp. 116-122, 1998.

[36] W. Ogryczak and T. Śliwiński, "On efficient WOWA optimization for decision support under risk," International Journal of Approximate Reasoning, vol. 50, no. 6, pp. 915-928, 2009.

[37] S. Wasserman and K. Faust, Social Network Analysis: Methods and Applications, Cambridge University Press, 1994.

[38] R. R. Yager, "On the cardinality index and attitudinal character of fuzzy measures," International Journal of General Systems, vol. 31, no. 3, pp. 303-329, 2002.

[39] R. Fullér and P. Majlender, "An analytic approach for obtaining maximal entropy OWA operator weights," Fuzzy Sets and Systems, vol. 124, no. 1, pp. 53-57, 2001.

[40] Y.-M. Wang and C. Parkan, "A minimax disparity approach for obtaining OWA operator weights," Information Sciences, vol. 175, no. 1-2, pp. 20-29, 2005.

[41] Y.-M. Wang, Y. Luo, and X. Liu, "Two new models for determining OWA operator weights," Computers \& Industrial Engineering, vol. 52, no. 2, pp. 203-209, 2007.

[42] Y. P. Jiang and Z. P. Fan, Decision Theory and Method Based on Judgement Matrix, Science Press, 2008, http://www.sciencep .com/s_single.php?id=16934. 


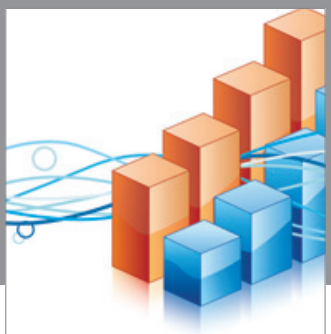

Advances in

Operations Research

vatem alat4

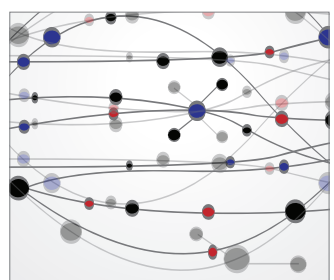

\section{The Scientific} World Journal
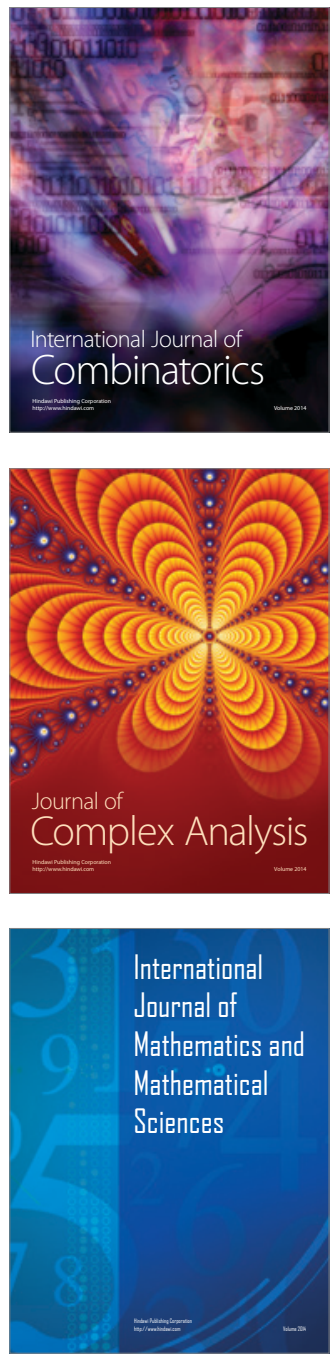
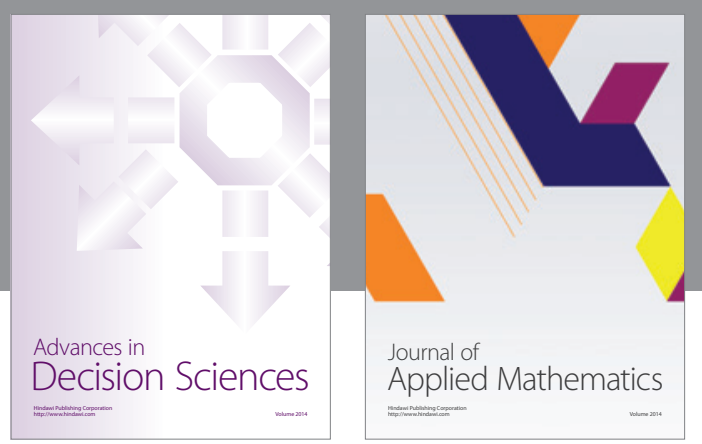

Algebra

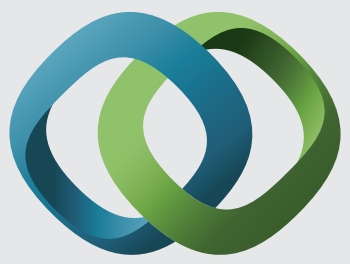

\section{Hindawi}

Submit your manuscripts at

http://www.hindawi.com
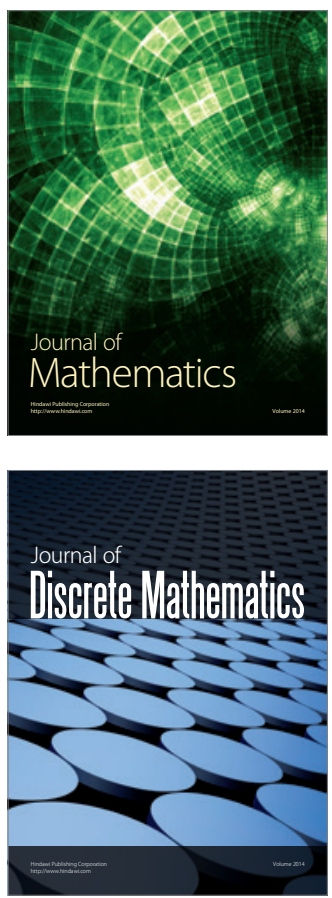

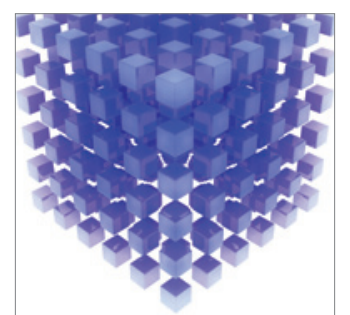

Mathematical Problems in Engineering
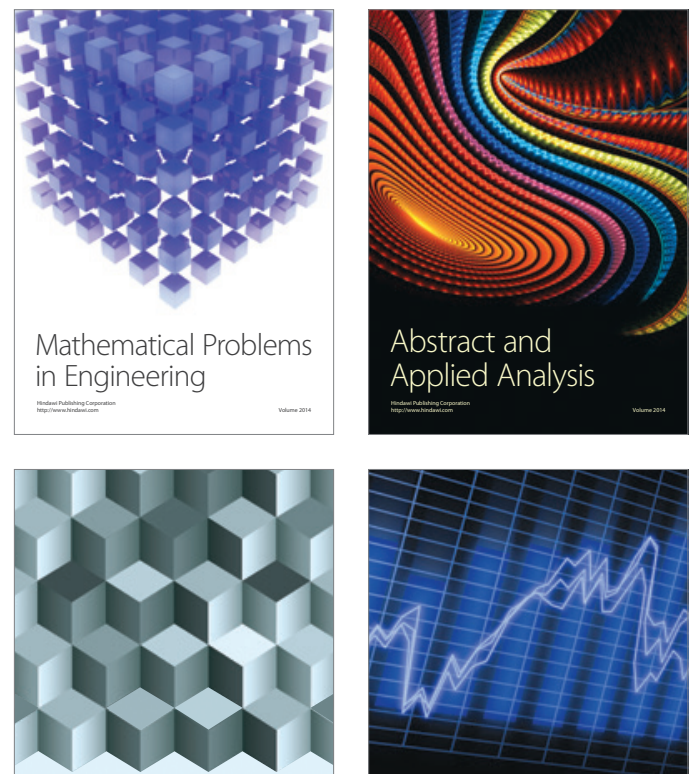

Journal of

Function Spaces

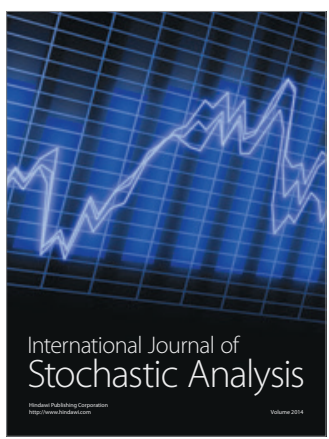

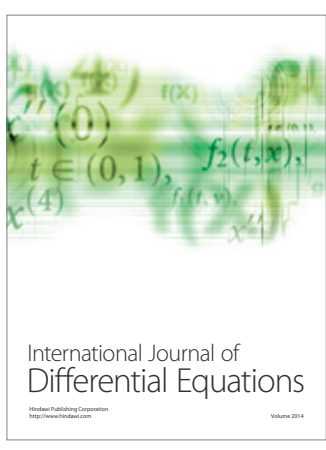
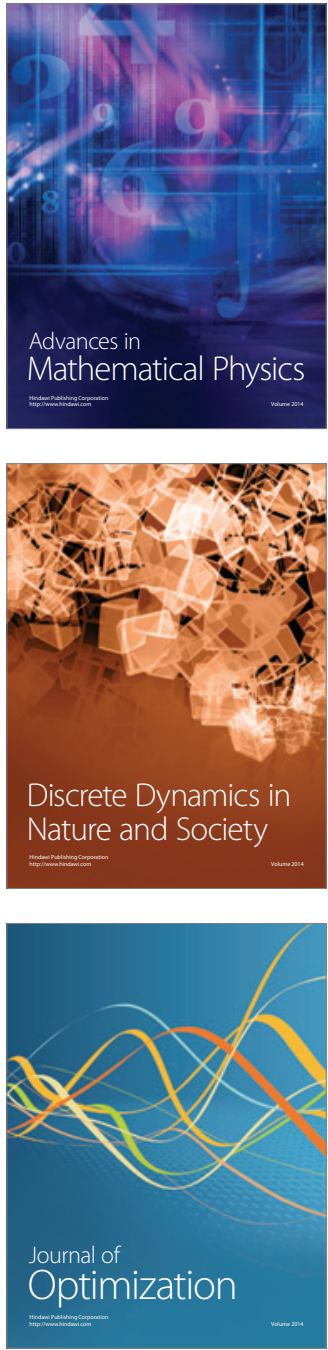